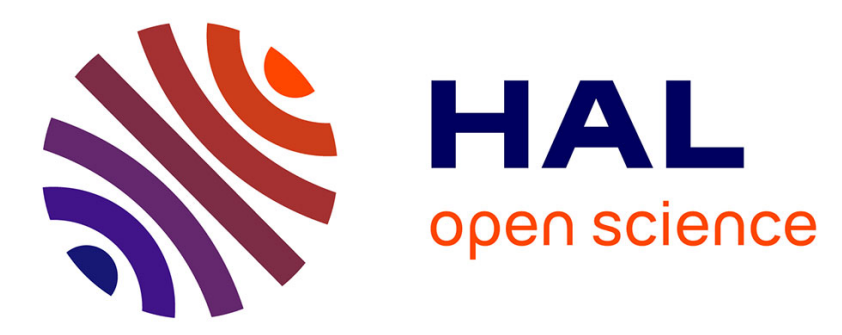

\title{
Optimal control of a crop irrigation model under water scarcity
}

Kenza Boumaza, Nesrine Kalboussi, Alain Rapaport, Sébastien Roux, Carole Sinfort

\section{- To cite this version:}

Kenza Boumaza, Nesrine Kalboussi, Alain Rapaport, Sébastien Roux, Carole Sinfort. Optimal control of a crop irrigation model under water scarcity. Optimal Control Applications and Methods, 2021, 42 (6), pp.1612-1631. 10.1002/oca.2749 . hal-03226630

\section{HAL Id: hal-03226630 https://hal.inrae.fr/hal-03226630}

Submitted on 14 May 2021

HAL is a multi-disciplinary open access archive for the deposit and dissemination of scientific research documents, whether they are published or not. The documents may come from teaching and research institutions in France or abroad, or from public or private research centers.
L'archive ouverte pluridisciplinaire HAL, est destinée au dépôt et à la diffusion de documents scientifiques de niveau recherche, publiés ou non, émanant des établissements d'enseignement et de recherche français ou étrangers, des laboratoires publics ou privés.

\section{(c)(1)}

Distributed under a Creative Commons Attribution| 4.0 International License 


\section{Introduction}

Today, in the context of the climate crisis, tensions around water resources are growing, and agriculture in many countries, particularly in the South, must now be considered as having to be water-saving. One way to cope with this changing context is to impose irrigation quotas. But such quotas could imply yield losses as the best crop water requirements would not necessarily be fulfilled. In these particular but expected situations, optimizing the schedule of irrigation to minimize yield losses becomes crucial. Crop modeling and numerical simulations are handy tools for understanding and adjusting these compromises. More particularly the control theory as irrigation is typically a control variable of a crop model.

Numerous texts and articles have developed systematic approaches to tackle the scheduling optimization problem. We refer to $[11,12,18,19,23]$ and references therein. However, most of the existing approaches do not allow to have analytical descriptions of an optimal solution's theoretical properties as they are based on the numerical optimization of complex models. Control theoretical tools, such as the Pontryagin's Maximum Principle, allow, as a rule, to discover structures of optimal strategies in terms of state feedback available for large classes of operating conditions and parameter values. Unlike optimization among open-loop controls that apply for precise requirements, closed-loop procedures offer adaptability and robustness for concrete applications, when state variables can be measured in real-time. However, when models have too numerous equations, variables, and parameters or do not present a robust structure, the study of optimal strategies with these techniques is most of the time out of reach of analytical characterizations. This is a motivation to consider models with a reasonable simplicity to benefit from these theoretical approaches (see e.g. [13, 17]). Even when these models are imperfect, optimal policies' derivation is of possible relevance. It generally provides simple but non-intuitive control rules that can be tested in simulation on more realistic detailed models. Sometimes, combinations of analytical description and reduced optimization can be obtained, which still offer advantages over purely numerical solutions in open-loop. This is the spirit in which this work has been conducted.

The aim of this work is investigate optimal irrigation strategies in the context of water quotas, with the help of a simplified crop model. The objective is to characterize the maximal biomass production that can be obtained for a given value of the quota (as an intrinsic performance of the system without consideration of water pricing), and for which feedback strategy it can be achieved. To the best of our knowledge, the analytical study of this

*corresponding author 
problem has not been yet addressed in the literature, even for simple stress functions. The present work goes two steps further than the preliminary one presented in [10], first in relaxing assumptions on the water stress functions of the model, and secondly in providing the complete solution of the optimal control problem as well as associated numerical simulations.

The organization of the paper is as follows. Section 2 presents the crop model with its assumptions. Section 3 is dedicated to the formulation of the optimal control problem under constraint, along with some preliminary results. In section 4, crucial properties of the optimal solutions are proved. Then, Section 5 is devoted to the application of the Pontryagin Maximum Principle and the synthesis of the optimal irrigation strategy. Finally, in Section 6 , we illustrate the theoretical results on numerical simulations and draw comparisons of several control strategies.

\section{Model description and assumptions}

We consider the dynamical model of crop irrigation introduced in [10] and inspired from [15], where $S(t)$ and $B(t)$ stand respectively for the relative soil humidity in the root zone (a quantity between 0 and 1 ) and the crop biomass at time $t$ in an interval $[0, T]$ representing the crop growth season, where 0 and $T$ are the sowing and harvesting dates. The control variable $u(t)=F(t) / F_{\max } \in[0,1]$ is the ratio of the input water flow rate $F(t)$ at time $t$ over the maximal flow $F_{\max }$ that the irrigation allows. In this model as in [1], crop evapotranspiration is split into crop transpiration $\varphi(t) K_{S}(S)$ and soil evaporation $(1-\varphi(t)) K_{R}(S)$ using the crop radiation interception efficiency $\varphi(t)$. The two functions $K_{S}$ and $K_{R}$ (see Assumption 1a and Fig. 1 below) are used to model the regulation of transpiration and evaporation by soil moisture as in [15].

Assumption 1a. The functions $K_{S}$ and $K_{R}$ are piecewise linear non decreasing from $[0,1]$ to $[0,1]$ with numbers $0<S_{h}<S_{w}<S^{\star}<1$ such that

1. $K_{S}$, resp. $K_{R}$ is null on $\left[0, S_{w}\right]$, resp. $\left[0, S_{h}\right]$, and positive outside this interval.

2. $K_{S}$ is equal to 1 on $\left[S^{\star}, 1\right]$ and concave increasing on $\left[S_{w}, S^{\star}\right]$.

3. $K_{R}(1)=1$ and $K_{R}$ is convex increasing on $\left[S_{w}, S^{\star}\right]$.

The value $S_{w}$ represents the plant wilting point, which is usually higher than the hydroscopic point denoted by $S_{h} . S^{\star}$ is the minimal threshold on the soil humidity that gives the best biomass production. This assumption generalizes the expressions found in the literature (see for instance [15]), given by the following assumption (see Fig. 1).

Assumption 1b. The functions $K_{S}$ and $K_{R}$ are piecewise linear non decreasing from $[0,1]$ to $[0,1]$ given by the following expressions

$$
K_{S}(S)=\left\{\begin{array}{cl}
0 & S \in\left[0, S_{w}\right], \\
\frac{S-S_{w}}{S^{\star}-S_{w}} & S \in\left[S_{w}, S^{\star}\right], \\
1 & S \in\left[S^{\star}, 1\right],
\end{array} \quad K_{R}(S)=\left\{\begin{array}{cl}
0 & S \in\left[0, S_{h}\right], \\
\frac{S-S_{h}}{1-S_{h}} & S \in\left[S_{h}, 1\right],
\end{array}\right.\right.
$$

where $0<S_{h}<S_{w}<S^{\star}<1$.

The piecewise linear assumption is related to the practitioners knowledge characterizing thresholds of various stages, and also the choice of a simple representation with few parameters. We shall say that $S$ is a corner point of $K_{S}$, resp. $K_{R}$ when the function is non differentiable at $S$ (therefore $S^{\star}$ is a corner point of $K_{S}$ ).

Assumption 2. The function $\varphi$ is $C^{1}$ increasing with $\varphi(0) \geq 0$ and $\varphi(T) \leq 1$.

In many situations, one can take $\varphi(0)=0$ at sowing date and $\varphi(T)=1$ at harvesting date.

We shall also consider dilution rate coefficients $k_{1}, k_{2}$ related respectively to soil characteristics (porosity) and irrigation system.

Assumption 3. $k_{1}, k_{2}$ are positive parameters with $k_{2} \geq 1$.

Finally, we denote the function $f$ for the biomass growth rate of the crop in absence of water stress (as a function of $B$ ). 

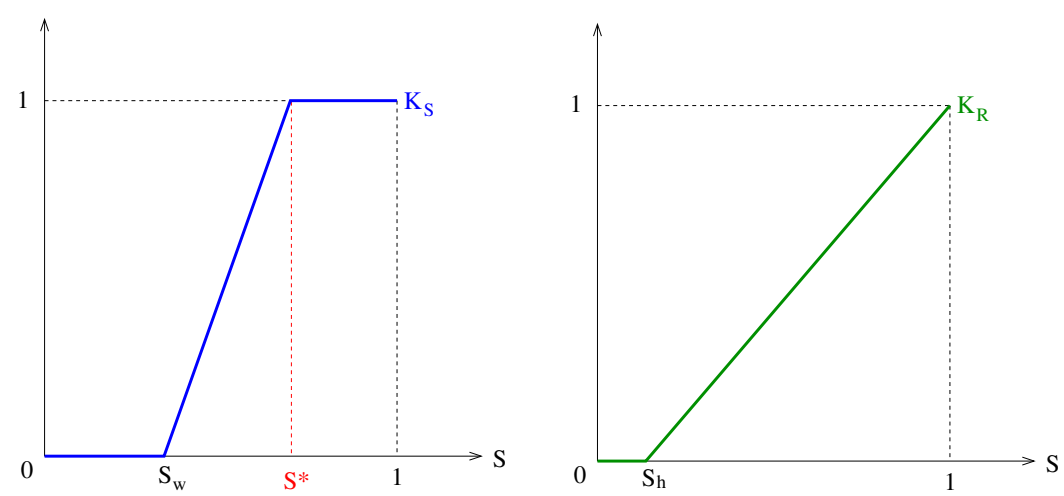

Figure 1: Graphs of the functions $K_{S}$ and $K_{R}$ given by expressions (1)

Assumption 4. The function $f$ is a non-negative Lipschitz continuous function with linear growth such that $f\left(B_{0}\right)>0$.

The equations of the model are then

$$
\begin{aligned}
\dot{S} & =k_{1}\left(-\varphi(t) K_{S}(S)-(1-\varphi(t)) K_{R}(S)+k_{2} u(t)\right) \\
\dot{B} & =\varphi(t) K_{S}(S) f(B)
\end{aligned}
$$

with initial condition

$$
\begin{aligned}
& S(0)=S_{0}>S^{\star} \\
& B(0)=B_{0}>0 .
\end{aligned}
$$

Eq. (2) represents the variation of a vertically averaged soil moisture as influenced by crop evapotranspiration $\varphi(t) K_{S}(S)+(1-\varphi(t)) K_{R}(S)$ and irrigation $k_{2} u(t)$. Eq. (3) determines the amount of biomass produced per time unit from the transpiration flux as in $[21,15]$ and modulated by a normalized growth kinetics function $f(\cdot)$. It is usually considered that crop is viable only if initial soil mixture $S_{0}$ is above the threshold $S^{\star}$. Indeed, $S_{0}$ is often equal to 1 at the chosen sowing date (typically at the end of winter season). Notice that the dynamics (2)-(3) is non-autonomous and that we consider a mild hypothesis on the function $\varphi$. The function $\varphi$, which makes the system non-autonomous, can be seen as a surrogate to the crop coefficient used in the FAO approach [24]. The condition $k_{2} \geq 1$ is a controllability assumption, in the sense that it allows the variable $S$ to stay equal to 1 with the constant control $u=1 / k_{2}$. Typical instances of growth function $f$ are constants or the logistic law, as considered in $[17]$

$$
f(B)=r B\left(1-\frac{B}{B_{\max }}\right),
$$

with $B_{\max }>B_{0}$, but other choices are possible. Note that the present model does not consider a temporal variation of the reference evapotranspiration present in many crop models and does not include either rainfall inputs: it would be more suitable for greenhouse-grown crops.

The dynamics is naturally subject to the state constraint

$$
S(t) \leq 1, \quad t \in[0, T]
$$

and we shall consider the set $\mathcal{U}$ of admissible controls as measurable functions $u(\cdot)$ taking value in $[0,1]$ such that the solution of (2)-(4) verifies the constraint (7). Under the former assumptions, one obtains straightforwardly the following property.

Lemma 1. For any admissible control $u(\cdot)$, the solution $(S(\cdot), B(\cdot))$ of system (2)-(3) with initial condition (4)-(5) verifies

$$
S(t)>S_{h}, \quad t \geq 0
$$

and $B(\cdot)$ is uniformly bounded on $[0, T]$. 
Remark 1. Another way to impose the state constraint (7) to be fulfilled is to consider that the extra water that could be brought when the soil is already saturated (i.e. $S=1$ ) is indeed lost. This amounts to consider the following dynamics of $S$ instead of equation (2):

$$
\dot{S}=k_{1}\left(-\varphi(t) K_{S}(S)-(1-\varphi(t)) K_{R}(S)+k_{2} \chi(S, u(t))\right),
$$

where the function $\chi(\cdot)$ is given by

$$
\chi(S, u):= \begin{cases}\min \left(1 / k_{2}, u\right) & \text { if } S=1, \\ u & \text { if } S<1 .\end{cases}
$$

Note that the right member of the ordinary differential equation (9) remains bounded, continuous w.r.t. ( $t, S, u)$ and Lipschitz in $S$, which then gives existence and uniqueness of solution of (9) for any measurable control function $u(\cdot)$ that takes values in $[0,1]$. However, we shall show in Section 4 (Proposition 2) that under water scarcity, an optimal solution never saturates this constraint, so that we no longer need to consider it.

\section{The optimization problem}

For each control $u(\cdot)$ in $\mathcal{U}$, we associate the total water delivered to the crop during the time interval $[0, T]$ as

$$
Q[u(\cdot)]:=F_{\max } \int_{0}^{T} u(t) d t
$$

and given a quantity of water $\bar{Q}>0$ available on the time interval $[0, T]$, we define the constraint

$$
Q[u(\cdot)] \leq \bar{Q}
$$

that defines the quota mentioned in the introduction. Then, the determination of the maximal biomass production is stated as the optimal control problem

$$
\sup \{B(T), u(\cdot) \in \mathcal{U} \text { that satisfies }(12)\} .
$$

By the usual argument of compactness of the set of admissible solutions, the dynamics being linear w.r.t. $u$ (see for instance [25]), one concludes about the existence of an optimal solution of Problem (13), that we aim now to characterize. Preliminary results are available in the conference paper [10], which are much proved and generalized in the present work.

Let $\bar{B}=B(T)$ where $B(\cdot)$ is solution of $\dot{B}=\varphi(t) f(B)$ with $B(0)=B_{0}$, which is a uniform bound of the solutions of $(3)-(5)$ on $[0, T]$. Note that $f$ is necessarily positive on $\left[B_{0}, \bar{B}\right)$ and one can consider the change of variable of the biomass

$$
B \mapsto \tilde{B}=g(B):=\int_{B_{0}}^{B} \frac{d b}{f(b)}, \quad B \in\left[B_{0}, \bar{B}\right),
$$

which gives the simplified dynamics

$$
\dot{\tilde{B}}=\varphi(t) K_{S}(S), \quad \tilde{B}=0
$$

instead of (3) and the true value of the biomass $B(t)$ can be recovered by $B(t)=g^{-1}(\tilde{B}(t))$, the function $g$ being increasing and thus invertible. For instance, for the logistic law (6), one has

$$
B(t)=\frac{B_{\max }}{1+\left(\frac{B_{\max }}{B_{0}}-1\right) e^{-r \tilde{B}(t)}}, \quad t \geq 0 .
$$

Therefore, maximizing $\tilde{B}(T)$ is equivalent to maximizing $B(T)$ (i.e. an optimal control maximizing $\tilde{B}(T)$ is also optimal for the problem (13)). For sake of simplicity, we shall drop the $f(B)$ term in equation (3) and consider $B_{0}=0$ without any loss of generality.

For convenience, we shall denote, for any $t_{s} \in[0, T]$ and $S_{s} \in[0,1], S_{t_{s}, S_{s}, 0}(\cdot)$, resp. $S_{t_{s}, S_{s}, 1}(\cdot)$, for the solution of the differential equation (2) with $S\left(t_{s}\right)=S_{s}$ and the constant control $u=0$, resp. $u=1$. We shall see in the following that the corner points, and especially the threshold $S^{\star}$, are playing a crucial role in the optimal synthesis. The following definitions will be useful in the following. 
Definition 1. Denote $\underline{S}(\cdot):=S_{0, S_{0}, 0}(\cdot)$ and define

$$
\underline{t}:=\sup \left\{t \in[0, T] \text { s.t. } \underline{S}(t)>S^{\star}\right\} .
$$

Define also the number

$$
B_{T}^{\star}:=\int_{0}^{T} \varphi(t) d t
$$

Straightforwardly, on has the first result.

\section{Lemma 2.}

(i) The inequality $B(T) \leq B_{T}^{\star}$ is fulfilled for any admissible control $u(\cdot)$.

(ii) If $\underline{t}=T$, then any admissible control $u(\cdot)$ gives $B(T)=B_{T}^{\star}$ (in particular the control identically null).

Let us now define singular controls as the ones that maintain $S(\cdot)$ constant.

Definition 2. For any $\tilde{S} \in(0,1)$, define the control

$$
\tilde{u}_{\tilde{S}}(t):=\frac{\varphi(t) K_{S}(\tilde{S})+(1-\varphi(t)) K_{R}(\tilde{S})}{k_{2}}, \quad t \in[0, T]
$$

and posit for $\tilde{S}=S^{\star}$

$$
Q^{\star}:=F_{\max } \int_{\underline{t}}^{T} \tilde{u}_{S^{\star}}(t) d t .
$$

Note that under Assumption 3, the control (14) is admissible i.e. one has $\tilde{u}_{\tilde{S}}(t) \in[0,1]$ at any $t \in[0, T]$ whatever is $\tilde{S} \in(0,1)$. Moreover one has

$$
\tilde{u}_{\tilde{S}}(t) \in(0,1), t \in(0, T), \tilde{S} \in\left(S_{h}, S^{\star}\right] .
$$

One can easily check that the following Lemma holds.

Lemma 3. Assume $\underline{t}<T$.

(i) For any $\bar{Q} \geq Q^{\star}$, the control

$$
u(t)= \begin{cases}0 & t \in[0, \underline{t}), \\ \tilde{u}_{S^{\star}}(t) & t \in[\underline{t}, T]\end{cases}
$$

satisfies the constraint (12) and gives $B(T)=B_{T}^{\star}$.

(ii) For any $\bar{Q}<Q^{\star}$ and admissible control $u(\cdot)$ satisfying the constraint (12), one has $B(T)<B_{T}^{\star}$.

Consequently, when $\underline{t}=T$ or $\bar{Q} \geq Q^{\star}$, we know that the maximal biomass production $B_{T}^{\star}$ can be reached with the control strategy (16) (other choices could be possible). We shall focus now on the complementary cases that fulfill the following conditions.

Hypothesis 1. $\underline{t}<T$ and $\bar{Q}<Q^{\star}$.

This hypothesis corresponds to situations of water scarcity, because there is not any enough water available to maintain the soil humidity constantly above or equal to the level $S^{\star}$ which provides the maximal production $B_{T}^{\star}$ at the harvesting time. Those situations are quite challenging from the control viewpoint because the crop has to suffer from dryness at a certain point and the question amounts to choose, to some extent, how and when, to impact as little as possible the biomass production at final time $T$. We start by investigating the behavior of the optimal solutions above the $S^{\star}$ level. 


\section{Properties of the optimal solutions with respect to threshold $S^{\star}$}

We introduce below the MRAP (for Most Rapid Approach Path) to $S=S^{\star}$ controls. Such kind of controls have already been considered in several optimal control problems in the plane, characterizing their optimality (e.g. $[14,8,7]$ or related to the so-called "turnpike" property (see e.g. [16, 22, 6]). Here, we use it in a different way. We do not pretend that these controls are necessarily optimal (and indeed they are not), but they respect the state constraint (7) and can locally improve the cost, providing then a comparison tool given in Proposition 1 below and used later on. We begin with some definitions.

Definition 3. For $\left(t_{s}, S_{s}\right) \in[0, T) \times\left(S^{\star}, 1\right]$, we define the number

$$
t^{+}\left(t_{s}, S_{s}\right)=\left\{\begin{array}{l}
T \quad \text { if } S_{t_{s}, S_{s}, 0}(t)>S^{\star}, t \in\left[t_{s}, T\right] \\
\inf \left\{t>t_{s} ; S_{t_{s}, S_{s}, 0}(t)=S^{\star}\right\} \text { otherwise }
\end{array}\right.
$$

And for any $\left(t_{s}, S_{s}\right) \in(0, T] \times\left(S^{\star}, 1\right]$, we define

$$
t^{-}\left(t_{s}, S_{s}\right)=\left\{\begin{array}{l}
0 \quad \text { if } S_{t_{s}, S_{s}, 1}(t)>S^{\star}, t \in\left[0, t_{s}\right] \\
\sup \left\{t<t_{s} ; S_{t_{s}, S_{s}, 1}(t)=S^{\star}\right\} \text { otherwise. }
\end{array}\right.
$$

Definition 4. For any $\left(t_{1}, S_{1}\right) \in[0, T) \times\left[S^{\star}, 1\right]$ and $\left(t_{2}, S_{2}\right) \in\left(t_{1}, T\right] \times\left[S^{\star}, 1\right]$ such that $S_{2}$ is attainable from $\left(t_{1}, S_{1}\right)$ at time $t_{2}$ with an admissible control, we associate the MRAP control $\tilde{u}(\cdot)$ on the time interval $\left[t_{1}, t_{2}\right]$ as follows:

i) If $t^{-}\left(t_{2}, S_{2}\right) \geq t^{+}\left(t_{1}, S_{1}\right)$ :

$$
\tilde{u}(t):= \begin{cases}0 & \text { if } t \in\left[t_{1}, t^{+}\left(t_{1}, S_{1}\right)\right) \\ \tilde{u}_{S^{\star}}(t) & t \in\left[t^{+}\left(t_{1}, S_{1}\right), t^{-}\left(t_{2}, S_{2}\right)\right] \\ 1 & \text { if } t \in\left(t^{-}\left(t_{2}, S_{2}\right), t_{2}\right] .\end{cases}
$$

ii) If $t^{-}\left(t_{2}, S_{2}\right)<t^{+}\left(t_{1}, S_{1}\right)$ :

$$
\tilde{u}(t):= \begin{cases}0 & \text { if } t \in\left[t_{1}, \bar{t}\left(t_{1}, S_{1}, t_{2}, S_{2}\right)\right) \\ 1 & \text { if } t \in\left(\bar{t}\left(t_{1}, S_{1}, t_{2}, S_{2}\right), t_{2}\right]\end{cases}
$$

where $\bar{t}\left(t_{1}, S_{1}, t_{2}, S_{2}\right)$ is the unique $\bar{t} \in\left[t_{1}, t_{2}\right]$ such that $S_{t_{1}, S_{1}, 0}(\bar{t})=S_{t_{2}, S_{2}, 1}(\bar{t})>S^{\star}$ (one can easily verify that the function $I(t):=S_{t_{1}, S_{1}, 0}(t)-S_{t_{2}, S_{2}, 1}(t)$ is decreasing on $\left[t_{1}, t_{2}\right]$ and such that $I\left(t_{1}\right) \geq 0, I\left(t_{2}\right) \leq 0$, which gives the existence and uniqueness of $\left.\bar{t}\left(t_{1}, S_{1}, t_{2}, S_{2}\right)\right)$.

These particular trajectories are depicted on Fig. 2 and 3.

Then, one has the following comparison result.

Proposition 1. Let $S(\cdot)$ be a solution of $(2)$ on $\left[t_{1}, t_{2}\right]$ (with $0 \leq t_{1}<t_{2} \leq T$ ) for an admissible control $u(\cdot)$ such that $S(t) \geq S^{\star}$ for any $t \in\left[t_{1}, t_{2}\right]$. Denote $S_{1}=S\left(t_{1}\right)$ and $S_{2}=S\left(t_{2}\right)$. Then, the solution $\tilde{S}(\cdot)$ of $(2)$ on $\left[t_{1}, t_{2}\right]$ with $\tilde{S}\left(t_{1}\right)=S_{1}$ and the MRAP control $\tilde{u}(\cdot)$ (given in Definition 4 ) satisfies the following properties:

$$
\begin{gathered}
\tilde{S}\left(t_{2}\right)=S_{2} . \\
\tilde{S}(t) \leq S(t), \quad t \in\left[t_{1}, t_{2}\right] . \\
\int_{t_{1}}^{t_{2}} \tilde{u}(t) d t \leq \int_{t_{1}}^{t_{2}} u(t) d t .
\end{gathered}
$$

Moreover, the last inequality is strict when $S(\cdot)$ and $\tilde{S}(\cdot)$ are not identical.

Proof. By construction, the solution $\tilde{S}(\cdot)$ verifies $\tilde{S}\left(t_{1}\right)=S\left(t_{1}\right)$ and $S\left(t_{2}\right)=S\left(t_{2}\right)$. Thus, property (18) is verified.

From standard comparison results of scalar differential equation with right hand sides that are Lipschitz continuous w.r.t. the state variable (see e.g. [26]), one has for any solution $S(\cdot)$ of (2) with $S\left(t_{s}\right)=S_{s}$ and any admissible control function $u(\cdot)$, the following frame

$$
S_{t_{s}, S_{s}, 0}(t) \leq S(t) \leq S_{t_{s}, S_{s}, 1}(t), \quad t \in\left[t_{s}, T\right]
$$




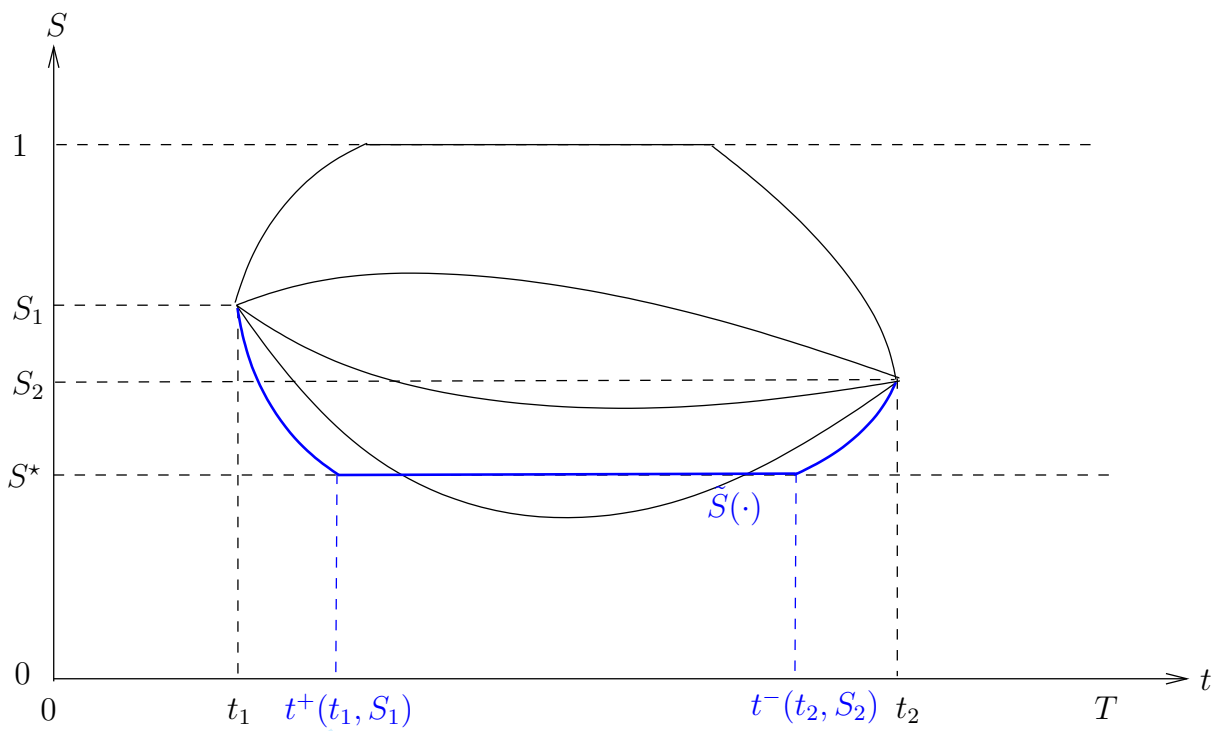

Figure 2: The MRAP trajectory $\tilde{S}(\cdot)$ (in blue) compared to other admissible trajectories $S(\cdot)$ when $t_{-}\left(t_{2}, S_{2}\right)>$ $t_{+}\left(t_{1}, S_{1}\right)$

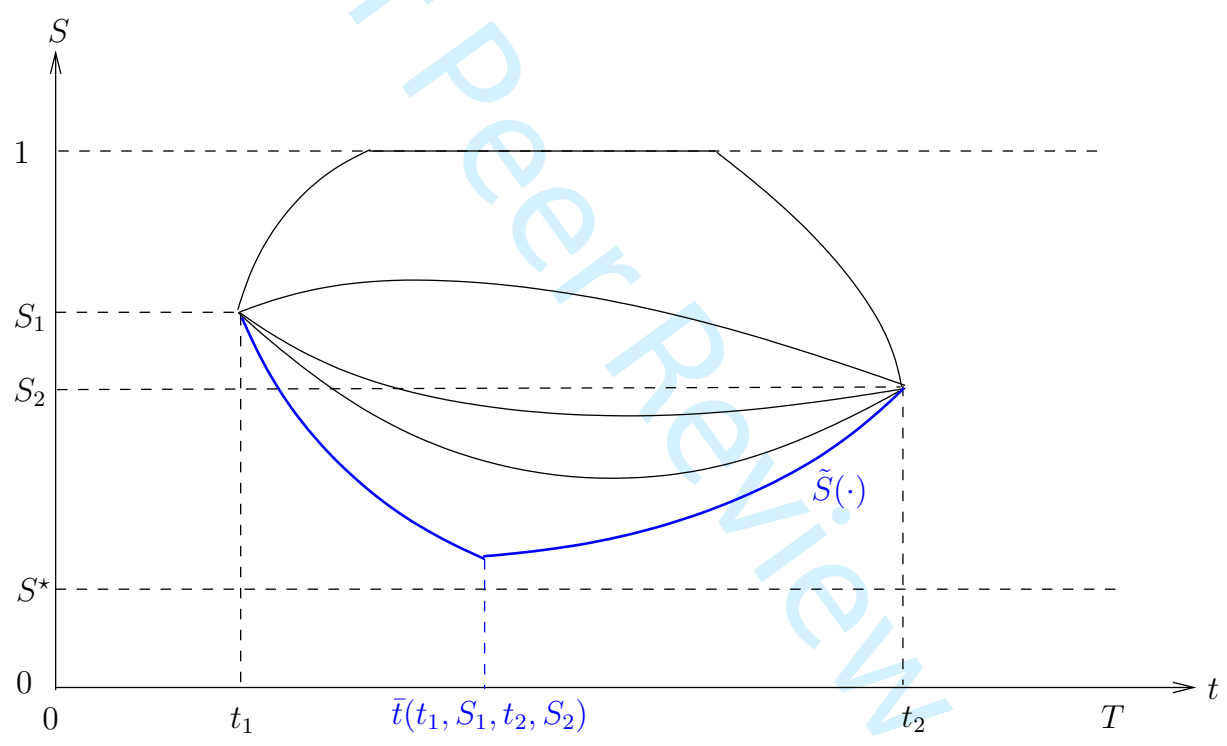

Figure 3: The MRAP trajectory $\tilde{S}(\cdot)$ (in blue) compared to other admissible trajectories $S(\cdot)$ when $t_{-}\left(t_{2}, S_{2}\right)<$ $t_{+}\left(t_{1}, S_{1}\right)$

Therefore, property (19) is verified.

Consider then the function $\delta(t):=S(t)-\tilde{S}(t)$. From expression (2), one can write

$$
d \delta=-k_{1}(F(t, S(t))-F(t, \tilde{S}(t))) d t+k_{1} k_{2}(u(t)-\tilde{u}(t)) d t
$$

where we posit

$$
F(t, S)=\varphi(t) K_{S}(S)+(1-\varphi(t)) K_{R}(S)
$$


Integrating (22) between $t=t_{1}$ and $t=t_{2}$, one obtains

$$
\begin{aligned}
\delta\left(t_{2}\right)-\delta\left(t_{1}\right)= & -k_{1} \int_{t_{1}}^{t_{2}}(F(t, S(t))-F(t, \tilde{S}(t))) d t \\
& +k_{1} k_{2}\left(\int_{t_{1}}^{t_{2}} u(t) d t-\int_{t_{1}}^{t_{2}} \tilde{u}(t) d t\right) .
\end{aligned}
$$

As $F$ is non-decreasing w.r.t. $S$ and $S(t) \geq \tilde{S}(t)$ for $t \in\left[t_{1}, t_{2}\right]$, one obtains

$$
\int_{t_{1}}^{t_{2}} u(t) d t-\int_{t_{1}}^{t_{2}} \tilde{u}(t) d t \geq \frac{\delta\left(t_{2}\right)-\delta\left(t_{1}\right)}{k_{1} k_{2}}=0
$$

which proves property $(20)$.

This result leads to the following properties of the optimal solutions.

Proposition 2. Assume that Hypothesis 1 is satisfied (water scarcity). Then, any optimal solution satisfies the following properties.

(i) $u(t)=0$ for a.e. $t \in[0, \underline{t}]$

(ii) $S(t) \leq S^{\star}$ for any $t \in[\underline{t}, T]$,

(iii) $Q[u(\cdot)]=\bar{Q}$.

Proof. Let $\tilde{u}(\cdot)$ be the MRAP control for $\left(t_{1}, S_{1}\right)=\left(0, S_{0}\right)$ and $\left(t_{2}, S_{2}\right)=\left(T, S^{\star}\right)$ (see Definition 4$)$.

Consider any $S(\cdot)$ solution of (2),(4) for an admissible control $u(\cdot)$ satisfying the constraint (12). Notice first that the set

$$
E:=\left\{t \in[0, T] \text { s.t. } S(t)<S^{\star}\right\}
$$

is non-empty, otherwise one would have $B(T)=B_{T}^{\star}$, which is excluded by Lemma 3.ii. Let $t^{\star}:=\inf E<T$. By continuity of $S(\cdot)$, one has necessarily $S\left(t^{\star}\right)=S^{\star}$ and by Proposition 1 (applied for $t_{1}<t_{2} \in\left[0, t^{\star}\right]$ ), one has

$$
\int_{0}^{t^{\star}} \tilde{u}(t) d t \leq \int_{0}^{t^{\star}} u(t) d t
$$

Notice that one has $\tilde{u}(t)=\tilde{u}_{S^{\star}}(t)$ for $t \in\left[t^{\star}, T\right]$. From Hypothesis 1 , the inequality

$$
Q[u(\cdot)]=F_{\max } \int_{0}^{T} u(t) d t<Q^{\star}=F_{\max } \int_{0}^{T} \tilde{u}(t) d t
$$

is fulfilled. Consequently, (23) and (24) give the inequality

$$
\int_{t^{\star}}^{T} u(t) d t<\int_{t^{\star}}^{T} \tilde{u}_{S^{\star}}(t) d t
$$

where $\tilde{u}_{S^{\star}}(t)<1$ for $t \in\left[t^{\star}, T\right)$ (cf property (15)). Therefore, the set

$$
E_{1}:=\left\{t \in\left[t^{\star}, T\right] \text { s.t. } u(t)<1\right\}
$$

is necessarily of non-null measure. Moreover, the set $E \cap E_{1}$ is also of non-null measure (otherwise one would have $u(t)=1$ for a.e. $t \in E$ that would imply that $S(\cdot)$ is increasing on $E$, which contradicts $\left.S\left(t^{\star}\right)=S^{\star}\right)$.

If $t^{\star}>\underline{t}$, inequality (23) is strict (by Proposition 1 applied on $\left[0, t^{\star}\right]$ ), and one can consider a control $v(\cdot)$ such that

$$
\left\{\begin{array}{lll}
v(t)=\tilde{u}(t), & & t \in\left[0, t^{\star}\right] \\
v(t)=u(t), & & t \in\left[t^{\star}, T\right] \backslash\left(E \cap E_{1}\right), \\
v(t) \in[u(t), 1], & & t \in E \cap E_{1}
\end{array}\right.
$$

with

$$
0<\int_{E \cap E_{1}}(v(t)-u(t)) d t \leq \int_{0}^{t^{\star}}(u(t)-\tilde{u}(t)) d t
$$


Then, one has

$$
Q[v(\cdot)] \leq Q[u(\cdot)] \leq \bar{Q}
$$

which guarantees that $v(\cdot)$ satisfies the constraint (12). Its associated solution $S_{v}(\cdot), B_{v}(\cdot)$ satisfies then $S_{v}(t) \geq$ $S(t)$ for any $t \in[0, T]$ with

$$
\int_{E \cap E_{1}} S_{v}(t) d t>\int_{E \cap E_{1}} S(t) d t
$$

As $S(t)<S^{\star}$ for $t \in E \cap E_{1}$, one obtains under Assumption 1a the inequality

$$
\int_{E \cap E_{1}} \varphi(t) K_{S}\left(S_{v}(t)\right) d t>\int_{E \cap E_{1}} \varphi(t) K_{S}(S(t)) d t
$$

which yields

$$
B_{v}(T)=\int_{0}^{T} \varphi(t) K_{S}\left(S_{v}(t)\right) d t>\int_{0}^{T} \varphi(t) K_{S}(S(t))=B(T) .
$$

We conclude that an optimal solution has to verify $t^{\star}=\underline{t}$, that is such that

$$
S(t)=\underline{S}(t), \quad t \in[0, \underline{t}]
$$

or equivalently that $u(t)=0$ for $t \in[0, t]$ is optimal.

Consider now a solution $S(\cdot), B(\cdot)$ with an admissible control $u(\cdot)$ that is null on $[0, t]$ and satisfies the constraint (12), and the set

$$
F:=\left\{t \in[\underline{t}, T] \text { s.t. } S(t)>S^{\star}\right\}
$$

is non empty. From Proposition 1, one has

$$
\int_{F} \tilde{u}(t) d t<\int_{F} u(t) d t
$$

Let us consider an admissible control $v(\cdot)$ such that

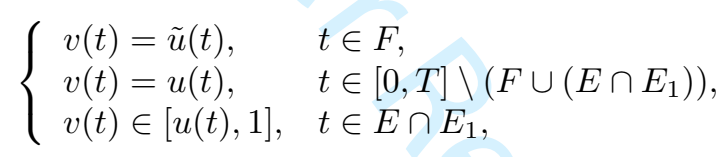

with

$$
0<\int_{E \cap E_{1}}(v(t)-u(t)) d t \leq \int_{F}(u(t)-\tilde{u}(t)) d t .
$$

Its solution $S_{v}(\cdot), B_{v}(\cdot)$ satisfies $S_{v}(t)=S^{\star}$ for $t \in F$ and $S_{v}(t) \geq S^{\star}$ for $t \in[0, T] \backslash F$ with

$$
\int_{E \cap E_{1}} S_{v}(t) d t>\int_{E \cap E_{1}} S(t) d t
$$

As before, we obtain inequalities (25), (26), and conclude that an optimal solution has to verify $F=\emptyset$, that is such that $S(t) \leq S^{\star}$ for $t \in[\underline{t}, T]$.

Finally, consider an admissible control $u(\cdot)$ that is null on $[0, \underline{t}]$ with $S(t) \leq S^{\star}$ for $t \in[\underline{t}, T]$ and $Q[u(\cdot)]<\bar{Q}$. As previously, one can consider another admissible control $v(\cdot)$ such that:

$$
\begin{cases}v(t)=u(t), & t \in[0, T] \backslash\left(E \cap E_{1}\right), \\ v(t) \in[u(t), 1], & t \in E \cap E_{1},\end{cases}
$$

with

$$
0<F_{\max } \int_{E \cap E_{1}}(v(t)-u(t)) d t \leq \bar{Q}-Q[u(\cdot)] .
$$

Its solution $S_{v}(\cdot), B_{v}(\cdot)$ satisfies $S_{v}(t) \geq S(t)$ for $t \in[0, T]$ with

$$
\int_{E \cap E_{1}} S_{v}(t) d t>\int_{E \cap E_{1}} S(t) d t
$$

One obtains again inequality (26), which shows that the control $u(\cdot)$ cannot be optimal. Therefore, an optimal control $u(\cdot)$ has to satisfy $Q[u(\cdot)]=\bar{Q}$. 


\section{Optimal synthesis}

Note first that one can write equivalently the optimization problem (13) as a (non-autonomous) scalar optimal control problem

$$
\max _{u(\cdot)} \int_{0}^{T} \varphi(t) K_{S}(S(t)) d t
$$

where $S(\cdot)$ is solution of (2), under constraints (7) and (12), or equivalently as an optimal control in the plane for the dynamics

$$
\begin{aligned}
\dot{S} & =k_{1}\left(-\varphi(t) K_{S}(S)-(1-\varphi(t)) K_{R}(S)+k_{2} u(t)\right), \quad S(0)=S_{0}, \\
\dot{V} & =u(t), \quad V(0)=0,
\end{aligned}
$$

with the target

$$
V(T) \leq \bar{V}:=\frac{\bar{Q}}{F_{\max }}
$$

and the criterion (27). Moreover, we know from Proposition 2 that under Hypothesis 1 the state constraint (7) is never saturated for any optimal solution.

\subsection{Application of the Maximum Principle}

Let us write the Hamiltonian associated to this optimal control problem:

$$
\begin{aligned}
& H\left(t, S, \lambda_{S}, \lambda_{V}, u\right):= \\
& \quad \lambda_{S} k_{1}\left(k_{2} u-\left(\varphi(t) K_{S}(S)+(1-\varphi(t)) K_{R}(S)\right)\right)+\lambda_{V} u+\lambda_{0} \varphi(t) K_{S}(S)
\end{aligned}
$$

and its adjoint equations:

$$
\begin{aligned}
& \dot{\lambda}_{S} \in \varphi(t)\left(\lambda_{S} k_{1}-\lambda_{0}\right) \partial_{C} K_{S}(S(t))+(1-\varphi(t)) \lambda_{S} k_{1} \partial_{C} K_{R}(S(t)), \\
& \dot{\lambda}_{V}=0
\end{aligned}
$$

where $\partial_{C} K_{S}, \partial_{C} K_{R}$ denote the Clarke generalized gradients of the Lipschitz maps $K_{S}, K_{R}$. Therefore, $\lambda_{V}$ is constant. The (non-smooth) Maximum Principle of Pontryagin (see for instance [4]) states that for any optimal solution $S(\cdot), V(\cdot), u(\cdot)$, there exists an adjoint vector $\lambda(\cdot)=\left(\lambda_{S}(\cdot), \lambda_{V}(\cdot)\right)$ which is an absolutely continuous solution of the adjoint system (32)-(33) and a scalar $\lambda_{0}$ equal to 0 or 1 such that

$$
\lambda_{0}+\left|\lambda_{S}(t)\right|+\left|\lambda_{V}(t)\right| \neq 0, \quad t \in[0, T]
$$

which satisfy the transversality condition

$$
\lambda_{S}(T)=0, \quad \lambda_{V} \leq 0
$$

(remind that $S(T)$ is free and that $V(T)$ touches the boundary of the target $V \leq \bar{V}$ at terminal time by Proposition 2), along with the maximization condition

$$
H\left(t, S(t), \lambda_{S}(t), \lambda_{V}(t), u(t)\right)=\max _{v \in[0,1]} H\left(t, S(t), \lambda_{S}(t), \lambda_{V}(t), v\right), \text { a.e. } t \in[0, T] .
$$

Defining the switching function

$$
\phi(t):=\lambda_{S}(t) k_{1} k_{2}+\lambda_{V},
$$

the maximization (36) gives, for a.e. $t \in[0, T]$

$$
\begin{cases}u(t)=1 & \text { if } \phi(t)>0 \\ u(t) \in[0,1] & \text { if } \phi(t)=0, \\ u(t)=0 & \text { if } \phi(t)<0 .\end{cases}
$$

We first show that that an optimal solution cannot be abnormal.

Lemma 4. For any optimal solution, one has $\lambda_{0}=1$. 
Proof. If $\lambda_{0}=0$, the only solution of (32) for the terminal condition (35) is $\lambda_{S}(t)=0$ for $t \in[0, T]$. Moreover, the constant value of $\lambda_{V}$ has to be negative to fulfill the conditions (35) and (34). This implies that $\phi(t)$ is negative for any $t \in[0, T]$ and by $(38)$, one has $u(t)=0$ for a.e. $t \in[0, T]$ i.e. $\underline{S}(\cdot)$ is the optimal trajectory. Let $\underline{t} \in[0, T]$ be such that $\underline{S}(\underline{t})=S^{\star}$. Then the control $v(\cdot)$ defined by

$$
\bar{v}(t)=\left\{\begin{array}{cc}
0, & t \in[0, \underline{t}), \\
\frac{\bar{Q}}{T-\underline{t}}, & t \in[\underline{t}, T],
\end{array}\right.
$$

is admissible, and its associated solution $S_{v}(\cdot)$ verifies

$$
S_{v}(t)=\underline{S}(t), t \in[0, \underline{t}), \quad S_{v}(t)>\underline{S}(t), t \in[\underline{t}, T]
$$

which implies the inequality

$$
\int_{0}^{T} \varphi(t) K_{S}\left(S_{v}(t)\right) d t>\int_{0}^{T} \varphi(t) K_{S}(\underline{S}(t)) d t,
$$

and thus is a contradiction with the optimality of $\underline{S}(\cdot)$.

We prove now sign properties of the adjoint variables, that will play a crucial role in the following.

Proposition 3. For any optimal solution, one has $\lambda_{S}(t) \geq 0$ for any $t \in[0, T]$. Moreover, one has $\lambda_{V}<0$.

Proof. Let us consider the set

$$
E:=\left\{t \in[0, T) \text { s.t. } \lambda_{S}(t)<0\right\}
$$

and assume by contradiction that $E$ is non-empty. As one has $S(t)>S_{h}$ for any $t \in[0, T]$ (cf Lemma 1), and the functions $K_{S}$ and $K_{R}$ are respectively non-decreasing and increasing on $\left[S_{h}, 1\right]$ (by Assumption 1a), one obtains from equation (32) that $\lambda_{S}$ is non-increasing on $E$. Therefore, one has $\sup E=T$ and $\lambda_{S}(T)<0$, which is a contradiction with the transversality condition (35).

If $\lambda_{V}=0, \lambda_{S}$ can be null on a time interval only if $K_{S}(S)$ is constant on this time interval, according to the adjoint equation (32). Then, for a.e. $t$ such that $S(t) \in\left(S_{w}, S^{\star}\right)$, one has $\phi(t)>0$ and thus $u(t)=1$, which prevents the solution to go below $S^{\star}$, in contradiction with Hypothesis 1 . We conclude that $\lambda_{V}$ is negative.

Then, Proposition 3 and the transversality condition (35) imply that one has $\phi(T)<0$, which gives straightforwardly the following property of the optimal solutions.

Corollary 4. For any optimal solution, there exists $\bar{t}<T$ such that $u(t)=0$ for a.e. $t \in[\bar{t}, T]$. Moreover, one has $S(T)<S^{\star}$.

\subsection{Study of singular arcs}

Let us now study the possibilities of singular arcs (we recall that a singular arc is a part of an optimal trajectory such that the switching function $\phi$ remains equal to zero).

Proposition 5. A singular arc on a closed interval I not reduced to a singleton satisfies $S(t)=\tilde{S}, t \in I$ where $\tilde{S}$ is a corner point of $K_{S}$ or $K_{R}$ that belongs to $\left(S_{w}, S^{\star}\right]$.

Proof. We proceed in two steps. We first show that a singular arc can only occur at a corner point of the function $K_{S}$ or $K_{R}$, studying the switching function. Then, we show by contradiction that a singular arc can occur only at corner points above the plant wilting threshold $S_{w}$.

A singular arc occurs when the switching function $\phi$ is equal to zero on a closed interval $I$ of non-null measure. This amounts to have $\lambda_{S}$ constant equal to $\lambda_{S}^{\star}:=-\lambda_{v} /\left(k_{1} k_{2}\right)>0$ on such an interval. If $K_{S}$ and $K_{R}$ are differentiable at $S\left(t_{1}\right)$ with $t_{1} \in I, K_{S}^{\prime}(S(t))$ and $K_{R}^{\prime}(S(t))$ are constant equal to $K_{S}^{\prime}\left(S\left(t_{1}\right)\right)$ and $K_{R}^{\prime}\left(S\left(t_{1}\right)\right)$ on a neighborhood $\left(t_{1}-\epsilon, t_{1}+\epsilon\right)$ of $t_{1}$ (as the functions $K_{S}, K_{R}$ are piecewise linear by Assumption 1a). Then, from equation (32), one gets

$$
\varphi(t)\left[\left(\lambda_{S}^{\star} k_{1}-1\right) K_{S}^{\prime}\left(S\left(t_{1}\right)\right)-\lambda_{S}^{\star} k_{1} K_{R}^{\prime}\left(S\left(t_{1}\right)\right]=-\lambda_{S}^{\star} k_{1} K_{R}^{\prime}\left(S\left(t_{1}\right)\right), \quad t \in I \cap\left(t_{1}-\epsilon, t_{1}+\epsilon\right) .\right.
$$

From Lemma 1, one has $S\left(t_{1}\right)>S_{h}$ and thus $K_{R}^{\prime}\left(S\left(t_{1}\right)\right)>0$ (by Assumption 1a). Finally, as the function $\varphi$ is strictly increasing, we deduce that (39) cannot be fulfilled. We deduce that a singular arc can occur only for constant $S=\tilde{S}$ that are non-differential points of $K_{S}$ or $K_{R}$. 
Let us now show that it can occur only at corner points above $S_{w}$. From Proposition 2, we know that a singular arc cannot be optimal at values $\tilde{S}$ above $S^{\star}$, and from Lemma 1, that it cannot occur for $\tilde{S} \leq S_{h}$. From equation (32), one should have

$$
0 \in \varphi(t)\left(\lambda_{S}^{\star} k_{1}-\lambda_{0}\right) \partial_{C} K_{S}(\tilde{S})+(1-\varphi(t)) \lambda_{S}^{\star} k_{1} \partial_{C} K_{R}(\tilde{S}) \text { a.e. } t \in I
$$

At $\tilde{S} \in\left(S_{h}, S_{w}\right)$, one has $\partial_{C} K_{S}(\tilde{S})=\{0\}$ and any element in $\partial_{C} K_{R}(\tilde{S})$ is positive. Therefore condition (40) cannot be fulfilled. We show now that a singular arc with $\tilde{S}=S_{w}$ cannot be part of an optimal solution. If it is, let $I=\left[t_{1}, t_{2}\right]$ and define

$$
\delta_{I}:=\int_{I} u(t) d t=\int_{I} \tilde{u}_{S_{w}}(t) d t<t_{2}-t_{1} .
$$

From Corollary 4, one has $t_{2}<T$. If $S(t) \leq S_{w}$ for any $t>t_{2}$, one would have $B(T)=B\left(t_{1}\right)$. Consider then the control

$$
\check{u}(t)= \begin{cases}u(t), & t \in\left[0, t_{1}\right) \\ 1, & t \in\left[t_{1}, t_{1}+\delta_{I}\right), \\ 0, & t \in\left[t_{1}+\delta_{I}, T\right]\end{cases}
$$

and the associated solution $\check{S}(\cdot), \check{B}(\cdot)$. This control would be admissible (i.e. $Q[\check{u}(\cdot)] \leq Q[u(\cdot)]=\bar{Q})$, and one would have $\check{B}(T)>\check{B}\left(t_{1}+\delta_{I}\right)>\check{B}\left(t_{1}\right)=B(T)$ (because $\check{S}(t)>S_{w}$ for $\left.t \in\left[t_{1}, t_{1}+\delta I\right]\right)$. Then, the control $u(\cdot)$ would not be optimal. Therefore $S(\cdot)$ has to take values above $S_{w}$ in the time interval $\left[t_{2}, T\right]$ and from what precedes for a.e. $t>t_{2}$ with $S(t)>S_{w}$, either $\dot{S}(t)>0($ with $u(t)=1)$ or $\dot{S}(t)<0($ with $u(t)=0)$ or $\dot{S}(t)=0$ (with a singular control). Then, from Corollary 4 we deduce that there exists a sub-interval $J=\left[\bar{t}_{1}, \bar{t}_{2}\right] \subset\left(t_{2}, T\right]$ with $\bar{t}_{1}<\bar{t}_{2}$ such that $S(t)>S_{w}$ for any $t \in J$ and $u(t)=0$ for a.e. $t \in J$.

We now construct another control that gives the same biomass production but with a lower water consumption. Consider a control with $t^{\dagger} \in I$, defined as follows

$$
u^{\dagger}(t)= \begin{cases}0 & \text { if } t \in\left[t_{1}, t^{\dagger}\right) \\ 1 & \text { if } t \in\left[t^{\dagger}, t_{2}\right] \\ u(t) & \text { otherwise }\end{cases}
$$

Denote by $S^{\dagger}, B^{\dagger}$ the corresponding solution. For $t^{\dagger}=t_{1}$, one has $S^{\dagger}\left(t_{2}\right)>S_{w}$ and for $t^{\dagger}=t_{2}, S^{\dagger}\left(t_{2}\right)<S_{w}$. By the intermediate value theorem, there exists $t^{\dagger_{0}} \in\left(t_{1}, t_{2}\right)$ such that $S^{\dagger_{0}}\left(t_{2}\right)=S_{w}$. Then, one has $S^{\dagger_{0}}(t)<S_{w}$ at any $t \in\left(t_{1}, t_{2}\right)$ and $S^{\dagger_{0}}(t)=S(t)$ for $t \in[0, T] \backslash\left(t_{1}, t_{2}\right)$. Moreover one has $B^{\dagger_{0}}(t)=B(t)$ for any $t \in[0, T]$ (due to $\dot{B}(t)=\dot{B}^{\dagger_{0}}(t)=0$ at $\left.t \in\left(t_{1}, t_{2}\right)\right)$. As one has $S^{\dagger_{0}}\left(t_{1}\right)=S^{\dagger_{0}}\left(t_{2}\right)=S_{w}$, one gets from the integration of equation (2) on $I$ and $K_{R}$ increasing on $\left(S_{h}, S_{w}\right)$, the inequality

$$
\int_{I} u^{\dagger_{0}}(t) d t=\frac{1}{k_{2}} \int_{I}(1-\varphi(t)) K_{R}\left(S^{\dagger_{0}}(t)\right) d t<\frac{1}{k_{2}} \int_{I}(1-\varphi(t)) K_{R}\left(S_{w}\right) d t=\int_{I} u(t) d t .
$$

Therefore, the control $u^{\dagger_{0}}(\cdot)$ gives the same cost $B^{\dagger_{0}}(T)=B(T)$ but with a lower water consumption i.e. $Q\left[u_{0}^{\dagger}(\cdot)\right]<$ $Q[u(\cdot)]=\bar{Q}$.

Finally, we derive a new control that produces a better objective. Let $\delta=\bar{Q}-Q\left[u_{0}^{\dagger}(\cdot)\right]>0$ and consider the control

$$
u^{\#}(t)= \begin{cases}u^{\dagger_{0}}(t), & t \in\left[0, \bar{t}_{1}\right) \cup\left[\min \left(\bar{t}_{2}, \bar{t}_{1}+\delta\right), T\right] \\ 1, & t \in\left[\bar{t}_{1}, \min \left(\bar{t}_{2}, \bar{t}_{1}+\delta\right)\right)\end{cases}
$$

which satisfies also the constraint (12). Denote $S^{\#}(\cdot), B^{\#}(\cdot)$ the solution with the control $u^{\#}(\cdot)$. One has $S^{\#}\left(\bar{t}_{1}\right)=S\left(\bar{t}_{1}\right)$ and $B^{\#}\left(\bar{t}_{1}\right)=B\left(\bar{t}_{1}\right)$ and then $S^{\#}(t)>S(t)$ for any $t>\bar{t}_{1}$, which implies $B^{\#}(t)>B(t)$ for any $t>\bar{t}_{1}$ (because $K_{S}\left(S^{\#}(t)\right)>K_{S}((t))$ for $\left.t \in J\right)$. Therefore, the control $u(\cdot)$ cannot optimal, which shows that $\tilde{S}=S_{w}$ cannot be a singular arc.

\subsection{The SMS strategy}

For convenience, we denote sub-sets of corner points of $K_{S}$ or $K_{R}$ as below.

Definition 5. For any $S \in\left(S_{w}, S^{\star}\right]$, let $\mathcal{C}(S)$ be the set of corner points $\tilde{S} \geq S$ in $\left(S_{w}, S^{\star}\right]$ and $n(S)=$ card $\mathcal{C}(S)$. Define then the increasing sequence of corner points $\left\{\tilde{S}_{i}(S)\right\}_{i=1 \ldots n(S)}$ such that $\mathcal{C}(S)=\left\{\tilde{S}_{1}(S), \ldots, \tilde{S}_{n(S)}(S)\right\}$.

Note that for any $S \in\left(S_{w}, S^{\star}\right]$, the set $\mathcal{C}(S)$ is non empty. It contains at least $S^{\star}$ as the largest element, i.e. $\tilde{S}_{n(S)}(S)=S^{\star}$. We define now the saturated multiple shots (SMS) strategy. 
Definition 6. For $S_{m} \in\left(S_{h}, S^{\star}\right]$ and a sequence of non decreasing numbers $V_{i} \in(0, \bar{V}], i \in\left\{1, \ldots, n\left(S_{m}\right)\right\}$ with at least one equal to $\bar{V}$, define the time-varying feedback control

$$
\psi_{S_{m},\left\{V_{i}\right\}}^{S M S}(t, S, V):= \begin{cases}0 & \text { if } V=\bar{V} \text { or } S>S_{m} \text { with } V=0 \\ \tilde{u}_{S}(t) & \text { if } S=\tilde{S}_{i}\left(S_{m}\right) \text { for } i \in\left\{1, \ldots, n\left(S_{m}\right)\right\} \text { with } V<V_{i} \\ 1 & \text { otherwise. }\end{cases}
$$

Note that this control strategy is admissible because it guarantees $V(T) \leq \bar{V}$. This strategy consists of starting the irrigation when the moisture $S$ reaches an irrigation trigger threshold $\bar{S}_{m}$, with one or several stages. If the humidity rate $S(t)$ reaches a level $\tilde{S}_{i}\left(S_{m}\right)$ for some $i \in\left\{1, \ldots, n\left(S_{m}\right)\right\}$ the flow rate is saturated to maintain $S$ constant at this level value as long as the used volume $V(t)$ stays below the value $V_{i}$. This is what we call a "saturated shot". Note that if $V_{i}$ is too small or if $\tilde{S}_{i}\left(S_{m}\right)$ cannot be reached, there is no saturation of the flow rate for this value i.e. the trajectory does not present a step at this value. The generated trajectory has then at most $n\left(S_{m}\right)$ increasing saturated shots. This is why we call this feedback control a "saturated multiple shots" (see Section 6 for an illustration). Remark also that once $S(\cdot)$ has reached $S_{m}$ then $S(t) \leq S^{\star}$ for any future time. We now give our main result about the optimality of the SMS strategy.

Theorem 1. Under Assumptions 1a, 2, 3, 4 and Hypothesis 1, there exists a value $S_{m} \in\left(S_{h}, S^{\star}\right]$ and a sequence of non decreasing numbers $V_{i}, i=1, \ldots, n\left(S_{m}\right)$ with at least one equal to $\bar{V}$ such that the SMS feedback (41) with

$$
\int_{0}^{t_{M}} \psi_{S_{m},\left\{V_{i}\right\}}^{S M S}(t, S(t), V(t)) d t=\bar{V} \quad \text { for some } t_{M}<T
$$

is optimal.

Proof. Let $u(\cdot)$ be an optimal control, $S(\cdot), V(\cdot)$ the associated solution of (28), (29) and $\lambda_{S}(\cdot), \lambda_{V}$ the adjoint variables given by the Maximum Principle (see Section 5.1). Recall first that under Hypothesis $1, S(\cdot)$ satisfies $S(t) \geq \underline{S}(t)$ for any $t \in[0, T]$ with $S(t) \leq S^{\star}$ for $t \in[\underline{t}, T]$ (cf Proposition 2.ii).

As $u(t)=0$ for a.e. $t \in[0, \underline{t}]$ (Proposition 2.i), the switching function $\phi(\cdot)$ has to be non-positive on $[0, \underline{t}]$, or equivalently one should have $\lambda_{S}(t) \leq \lambda_{S^{\star}}=-\lambda_{v} /\left(k_{1} k_{2}\right)>0$ for $t \in[0, \underline{t}]$. On the interval $[0, \underline{t}], S(\cdot)$ is thus decreasing with $S(\underline{t})=S^{\star}$. As $K_{R}$ and $K_{S}$ are respectively increasing and constant on the interval [ $\left.S^{\star}, 1\right]$, we deduce from $(32)$ that $\lambda_{S}(t)<\lambda_{S^{\star}}$ for any $t \in[0, \underline{t})$.

Consider the set

$$
C:=\left\{t \in[\underline{t}, T] \text { s.t. } \lambda_{S}(t) \geq \lambda_{S^{*}}\right\}
$$

which is of non empty interior (otherwise $u(t)=0$ would be optimal for any $t \in[\underline{t}, T]$, which is not possible by Proposition 2.iii). Let us show that the set $C$ is connected. If not, consider a time interval $\left(t_{1}, t_{2}\right)$ in-between two consecutive connected components of $C$, that is such that $\lambda_{S}\left(t_{1}\right)=\lambda_{S}\left(t_{2}\right)=\lambda_{S^{*}}$ and $\lambda_{S}(t)<\lambda_{S^{\star}}$ for any $t \in\left(t_{1}, t_{2}\right)$. Note that $\lambda_{S}(t)<\lambda_{S}$ for $t \in\left(t_{1}, t_{2}\right)$ implies that $u(t)=0$ for almost any $t \in\left(t_{1}, t_{2}\right)$ and consequently $S(\cdot)$ is decreasing on $\left(t_{1}, t_{2}\right)$.

The function $\lambda_{S}$ attains necessarily its minimum on $\left(t_{1}, t_{2}\right)$, say at $\hat{t}$, and one has then

$$
\left.0 \in \varphi(\hat{t})\left(\lambda_{S}(\hat{t}) k_{1}-1\right) \partial_{C} K_{S}(S(\hat{t}))+(1-\varphi(\hat{t})) \lambda_{S}(\hat{t})\right) k_{1} \partial_{C} K_{R}(S(\hat{t})) .
$$

If $S(\hat{t})<S_{w}$, one has $\partial_{C} K_{S}(S(\hat{t}))=\{0\}$ and $\partial_{C} K_{R}(S(\hat{t})) \subset \mathbb{R}_{+}^{\star}$ (by Assumption 1a and Lemma 1), and thus (43) cannot be satisfied. So, one has necessarily $S(\hat{t}) \geq S_{w}$. Note that one has also $\lambda_{S}(\hat{t}) k_{1}-1<0$.

As $S(\cdot)$ is decreasing on $\left(t_{1}, t_{2}\right), K_{S}^{\prime}(S(t))$ and $K_{R}^{\prime}(S(t))$ exist for almost any $t \in\left(t_{1}, t_{2}\right)$. The function $K_{S}$, resp. $K_{R}$, being concave, resp. convex, on $\left(S_{w}, S^{\star}\right)$, and as $S(t) \in\left(S_{w}, S^{\star}\right)$ for any $t \in\left(t_{1}, \hat{t}\right)$, one has the property

$$
S(t)>S(\hat{t}) \Rightarrow\left\{\xi \geq K_{S}^{\prime}(S(t))>0, \forall \xi \in \partial_{C} K_{S}(S(\hat{t})) \text { and } K_{R}^{\prime}(S(t)) \geq \zeta>0, \forall \zeta \in \partial_{C} K_{R}(S(\hat{t}))\right\},
$$

for almost any $t \in\left(t_{1}, \hat{t}\right)$. Now, $\dot{\lambda}_{S}(t)$ exists for a.e. $t \in\left(t_{1}, \hat{t}\right)$ and one can write

$$
\begin{aligned}
\dot{\lambda}_{S}(t) & \left.=\varphi(t)\left(\lambda_{S}(t) k_{1}-1\right) K_{S}^{\prime}(S(t))+(1-\varphi(t)) \lambda_{S}(t)\right) k_{1} K_{R}^{\prime}(S(t)) \\
& \left.\geq \varphi(t)\left(\lambda_{S}(\hat{t}) k_{1}-1\right) K_{S}^{\prime}(S(t))+(1-\varphi(t)) \lambda_{S}(\hat{t})\right) k_{1} K_{R}^{\prime}(S(t)),
\end{aligned}
$$

for almost any $t \in\left(t_{1}, \hat{t}\right)$. Finally, as the function $\varphi$ is increasing and positive, one obtains with (44) the inequality

$$
\left.\dot{\lambda}_{S}(t) \geq \varphi(\hat{t})\left(\lambda_{S}(\hat{t}) k_{1}-1\right) \xi+(1-\varphi(\hat{t})) \lambda_{S}(\hat{t})\right) k_{1} \zeta, \quad \forall \xi \in \partial_{C} K_{S}(S(\hat{t})), \forall \zeta \in \partial_{C} K_{R}(S(\hat{t}))
$$


for almost any $t \in\left(t_{1}, \hat{t}\right)$. From (43), one gets $\dot{\lambda}_{S}(t) \geq 0$ for almost any $t \in\left(t_{1}, \hat{t}\right)$, which contradicts $\lambda_{S}(\hat{t})$ being a minimum of $\lambda_{S}$ on $\left(t_{1}, t_{2}\right)$. The set $C$ is thus connected.

The set $C$ being non-empty connected and $\lambda_{S}(\cdot)$ continuous, $C$ is a closed interval $\left[t_{m}, t_{M}\right]$ and $u(t)=0$ for a.e. $t \notin C$. From Proposition 2 and Lemma 1, we have that $t_{m} \geq \underline{t}$ and $S_{m}=S\left(t_{m}\right)$ belongs to $\left(S_{h}, S^{\star}\right]$. Moreover, from Corollary 4, we have $t_{M}<T$. Then, at any $t \in C$, the switching function $\phi(t)$ is non-negative. Therefore, for a.e. $t \in C$, either $u(t)=1$ or $u(t)=\tilde{u}_{\tilde{S}}(t)$ with $\tilde{S} \in\left(S_{w}, S^{\star}\right)$ a corner point (Proposition 5$)$ ). Consequently, $V(\cdot)$ is increasing on $C$ and $S(\cdot)$ non decreasing on $C$, composed of increasing parts $u=1$ and possibly singular ones $S=\tilde{S}_{i}$ with $\tilde{S}_{i} \in \mathcal{C}\left(S_{m}\right)$. As $V(\cdot)$ is increasing, the time $t_{i}$ when the solution $S(\cdot)$ leaves $S=\tilde{S}_{i}$ is equivalently defined by the value $V_{i}=V\left(t_{i}\right)$ reached by the variable $V(\cdot)$. If $\tilde{S}_{j}$ is not reached, we can set by convention $V_{j}=\bar{V}$.

At $t=t_{M}$, Proposition 2 gives $S\left(t_{M}\right) \leq S^{\star}$ and $V\left(t_{M}\right)=\bar{V}$. Then, either one has $S\left(t_{M}\right) \notin \mathcal{C}\left(S_{m}\right)$ and $S^{\star}=\tilde{S}_{n\left(S_{m}\right)}$ is not reached, or $S\left(t_{M}\right)=\tilde{S}_{j} \in \mathcal{C}\left(S_{m}\right)$ with $j \in\left\{1, \ldots, n\left(S_{m}\right)\right\}$ and $V_{j}=\bar{V}$. In any case there exists $i \in\left\{1, \ldots, n\left(S_{m}\right)\right\}$ such that $V_{i}=\bar{V}$. Finally, one can easily check that $u(\cdot)$ fulfills $u(t)=\psi_{S_{m},\left\{V_{i}\right\}}^{S M S}(t, S(t), V(t))$ for a.e. $t \in[0, T]$, and property iii of Proposition 2 imposes that the $S_{m}$ and the $V_{i}$ are such that the equality (42) is satisfied.

Let us underline that the structure of the feedback (41) does not depend on the shape of the radiation interception efficiency function $\varphi$, although the optimal switching times do depend on the values of this function.

\subsection{The SOS strategy}

When the functions $K_{S}, K_{R}$ have no corner on the interval $\left(S_{w}, S^{\star}\right)$, as it is the case for Assumption 1b, the SMS strategy takes a simpler expression, that we define as a "saturated one shot" (SOS) strategy as follows.

Definition 7. For $S_{m} \in\left(S_{h}, S^{\star}\right]$, define the time-varying feedback control

$$
\psi_{S_{m}}^{S O S}(t, S, V):= \begin{cases}0 & \text { if } V=\bar{V} \text { or } S>S_{m} \text { with } V=0, \\ \tilde{u}_{S^{\star}}(t) & \text { if } S=S^{\star} \text { with } V<\bar{V}, \\ 1 & \text { otherwise. }\end{cases}
$$

This strategy consists of irrigating crops at once when the humidity rate $S(t)$ gets equal to $S_{m}$. The water is delivered at the maximal flow rate $(u=1)$ as long as the humidity rate $S(\cdot)$ is below $S^{\star}$, or maintain it $S=S^{\star}$ (with the singular control $\tilde{u}_{S^{\star}}(\cdot)$ ), until the entire water budget $\bar{V}$ has been used up. This strategy is parameterized by the single value $S_{m}$ or equivalently the time when irrigation starts. This explains the wording "saturated one shot". One has the following result about the optimality of this strategy.

Theorem 2. Under Assumptions 1b, 2, 3, 4 and Hypothesis 1, there exists a value $S_{m} \in\left(S_{h}, S^{\star}\right]$ such that the SOS strategy (45) with

$$
\int_{0}^{t_{M}} \psi_{S_{m}}^{S O S}(t, S(t), V(t)) d t=\bar{V} \quad \text { for some } t_{M}<T
$$

is optimal.

Proof. Under Assumption 1b, one has $n(S)=1$ for any $S \in\left(S_{h}, S^{\star}\right)$ with $V_{1}=\bar{V}$. Therefore, the SMS feedback (41) has only one parameter $S_{m}$ which gives the simpler expression (45).

\section{Numerical simulations and discussion}

In this section, we compare numerically three irrigation strategies on two examples: the SMS and SOS previously introduced and the "One Shot" (OS) strategy which consists of delivering water at maximum flow rate during a single irrigation period at a triggering humidity level $S_{m}$.

Definition 8. For $S_{m} \in\left(S_{h}, S^{\star}\right]$, we denote by $t_{S}$ the irrigation triggering time associated to an humidity level $S_{m}\left(t_{S}=\underline{S}^{-1}\left(S_{m}\right)\right)$ and define the OS open-loop control as follows :

$$
u_{S_{m}}^{O S}(t):= \begin{cases}0 & \text { if } \left.t<t_{S} \text { or } t>\min \left(t_{S}+\bar{Q} / F_{\max }, T\right)\right), \\ 1 & \text { if } t \in\left[t_{S}, \min \left(t_{S}+\bar{Q} / F_{\max }, T\right)\right) .\end{cases}
$$


The OS strategy is a pure "bang-bang" control that is implemented in open-loop (once the irrigation starts, it lasts $\left.\left.\bar{Q} / F_{\max }\right)\right)$. It represents a class of widely used irrigation strategies, typically when drip irrigation is not available. For this strategy, we shall look for the triggering humidity level $S_{m}$ that give the best biomass production, to be compared with the biomass obtained with the best SMS and SOS strategies.

Theorem 1 has shown that the original optimization problem in infinite dimension is transformed into a simple finite dimensional optimization problems of parameters $\left(S_{m}, V_{1}, \ldots, V_{n}\right)$. Therefore, the optimal SMS (and SOS) strategy have been numerically determined, looking for the best parameters $\left(S_{m}, V_{1}, \ldots, V_{n}\right)$ in the set

$$
\mathcal{P}:=\left(S_{w}, S^{\star}\right] \times\left\{V \in \mathbb{R}^{n} ; 0 \leq V_{1} \leq V_{2} \leq \cdots \leq V_{n}=\bar{V}\right\}
$$

where $n \geq 1$ is the number of distinct corner points $\tilde{S}$ of the functions $K_{S}$ or $K_{R}$ in the interval $\left(S_{w}, S^{\star}\right]$. For each $\left(S_{m}, V_{1}, \ldots, V_{n}\right)$ in $\mathcal{P}$, the SMS feedback (41) is well defined and generates a unique solution of equations (2)-(3), to which we associate the cost $B(T)$, even though the constraint (42) might not be satisfied. Indeed, when looking for values of the parameters in $\mathcal{P}$ that give the largest $B(T)$, we know that the optimal ones necessarily satisfy the equality (42), according to Proposition 2.

In order to compare different irrigation strategies by their triggering level, we also introduce the partially optimized control SMS*, parameterized by a humidity level $S_{m}$, which corresponds to the best SMS strategy for a given triggering level $S_{m}$. This strategy can thus be obtained numerically by the approach presented above but optimizing with respect to the only parameters $\left(V_{1}, \ldots, V_{n}\right)$. This allows us the compare the crop productions provided by the three strategies SMS, SOS, OS for the same triggering level $S_{m}$. For each of them, their optimal production is then obtained for their best value $S_{m}$.

The performances of these strategies are compared for two kinds of configurations: one with the simplest $K_{s}, K_{r}$ functions that fulfill Assumption 1b, (see Fig. 1) and a second one when the function $K_{s}$ exhibits a more complex shape (see Fig. 4). For illustrative purposes only, we have considered dimensionless parameters (by normalizing the units), functions $\varphi$ in the family of $t \mapsto(t / T)^{\alpha}(\alpha>0)$ and functions $f$ as logistic laws $B \mapsto r B\left(1-\frac{B}{B_{m a x}}\right)$ parameterized by $\left(r, B_{\max }, B_{0}\right)$. The optimal solutions have been verified with the Bocop-HJB solver [2] that provided with a very good accuracy the same optimal trajectories, but in open-loop.

\subsection{Under Assumption 1b}

We recall that under this assumption, the SMS and SOS strategies coincide. We present in Fig. 5 the simulations performed with irrigation strategies SOS and OS and with inputs data given in Table 1.

\begin{tabular}{ccccccccccccc}
$T$ & $k_{1}$ & $k_{2}$ & $S^{\star}$ & $S_{w}$ & $S_{h}$ & $F_{\max }$ & $\bar{Q}$ & $\alpha$ & $r$ & $B_{\max }$ & $S_{0}$ & $B_{0}$ \\
\hline 1 & 2.1 & 5 & 0.7 & 0.4 & 0.2 & 1.2 & 0.1 & 4 & 25 & 1 & 1 & 0.0005 \\
\hline
\end{tabular}

Table 1: Normalized parameters used for the simulations under Assumption 1b

The best OS strategy was obtained for a triggering level $S_{m}=0.338$ and produced a biomass $B(T)=0.022$. The corresponding humidity dynamics are plotted in Fig. 5c. It can be seen that some values of $S$ are above $S^{\star}$. It can be therefore concluded from the application of Proposition 2 that an OS strategy cannot be optimal. This is further illustrated by applying the SOS strategies for the same input data. We find that the optimal SOS strategy gives a final biomass $B(T)=0.0388$ which is $77 \%$ higher than what gives the best OS strategy. The associated control is a bang-singular-bang (see Fig. 5b).

\subsection{Under Assumption 1a without Assumption 1b}

In this example, we consider that the function $K_{s}$ presents an additional corner point $S_{c}$ in between $S_{w}$ and $S^{\star}$ (see Fig. 4). We present in Fig. 6 the simulations performed with irrigation strategies SMS, SOS and OS and with inputs data given in Table 2. The biomass levels obtained when optimizing these strategies were 0.111 for SMS, 0.094 for SOS and 0.052 for OS. The best production is obtained with the SMS strategy (as expected by Theorem 1) and corresponds to the highest triggering humidity level $(S=0.43)$ among the three tested strategies (Fig. 6a). This level corresponds to the earliest irrigation triggering time $\left(t_{S}=0.392\right.$, see Fig. $\left.6 \mathrm{~b}\right)$.

We have shown that the optimal solution may have a singular arc corresponding to intermediate corner points. When looking at the humidity profile in Fig. 6c, we can see that indeed two arcs do occur in this setting: one at $S=S_{c}$ and another one later at $S=S^{\star}$. 

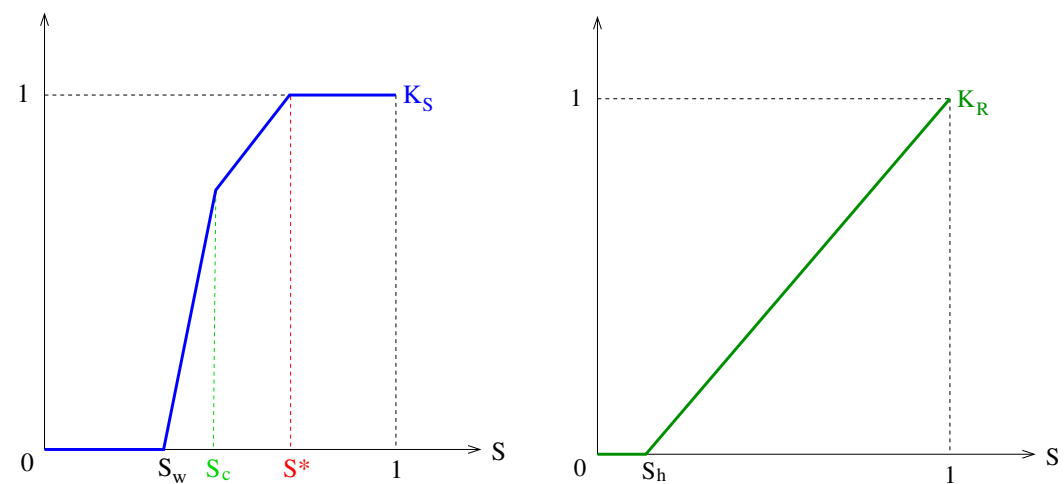

Figure 4: Graphs of the functions $K_{S}$ and $K_{R}$ that fulfill Assumption 1a but not Assumption 1b

\begin{tabular}{cccccccccccccc}
$T$ & $k_{1}$ & $k_{2}$ & $S^{\star}$ & $S_{w}$ & $S_{h}$ & $F_{\max }$ & $\bar{Q}$ & $\alpha$ & $r$ & $B_{\max }$ & $S_{0}$ & $B_{0}$ & $S_{c}$ \\
\hline 1 & 2.5 & 5 & 0.7 & 0.4 & 0.2 & 1.2 & 0.09 & 3 & 25 & 1 & 1 & 0.0005 & 0.43 \\
\hline
\end{tabular}

Table 2: Normalized parameters used for the simulations under Assumption 1a.

(a) Final biomass

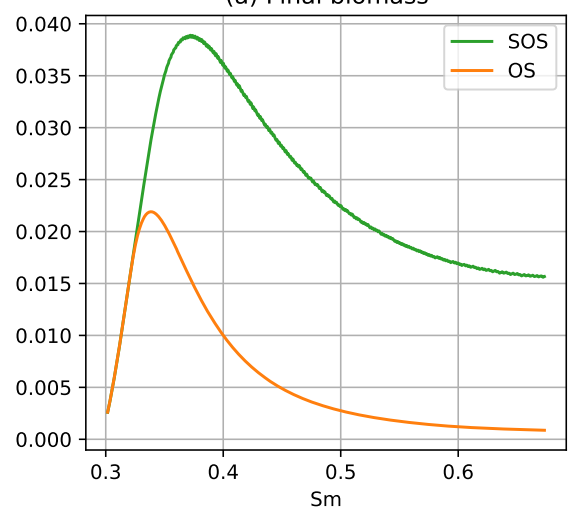

(b) Optimal Control

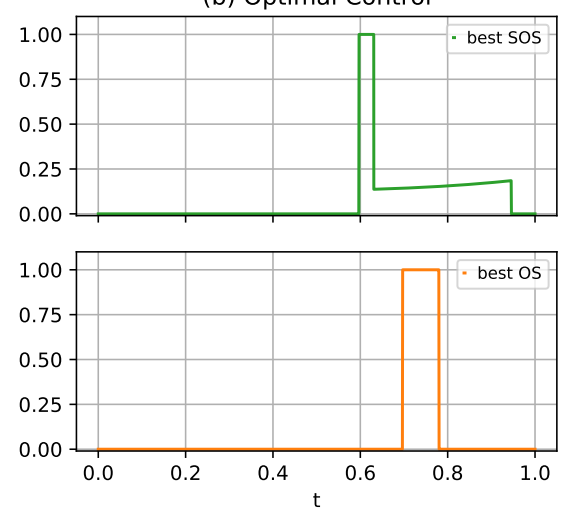

(c) Humidity

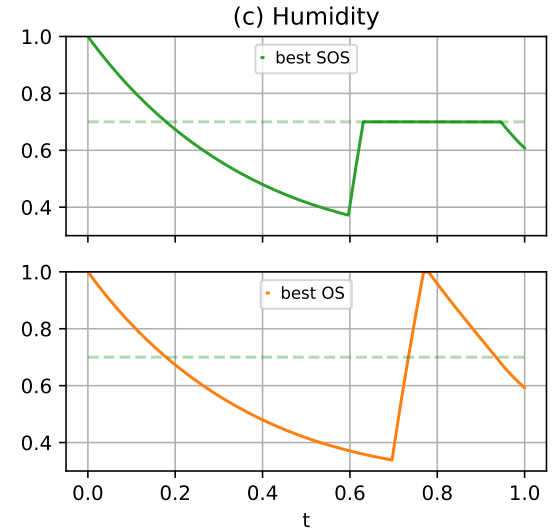

Figure 5: Comparison of OS and SOS controls strategies with model parameters given in Table 1.

\subsection{Discussion}

The structure of SMS strategies differs from the structure of solutions obtained by the authors in [17] with a comparable methodological approach but different model, criterion and constraint: their optimal irrigation policy consists of bringing the soil moisture from its initial level to an optimal target value as fast as possible and maintaining it until harvesting time when irrigation ceases. In a different context (with rainfall inputs and no water quota), the authors of [13] study on a simple irrigation model the optimal control minimizing the total quantity of water to ensure the soil humidity to remain above a given threshold $S_{\min }$. They found an optimal control policy whose structure is an SOS strategy with $\bar{S}=S_{\min }$ but with a triggering level equal to $\bar{S}$. We believe that these differences between these models, their criterion, and their optimal solutions are of interest and make our mathematical analysis worth of interest.

Note finally that the practical implementation of an SMS (OR SOS) strategy on a real irrigation system requires an adaptive controller to maintain the humidity level constant at the values of the singular arcs (differently to the OS strategy), which needs the on-line measurement of the variable S. Moreover, differently to the OS strategy, it gradually changes the input flow rate during the singular arc phase. Therefore, the SMS (or SOS) strategy can be considered more "sophisticated" than the OS one, as it requires a humidity sensor for its concrete application. Finally, this work allows to provide the best crop-water production functions (mapping water quotas to maximal 
Figure 6: Comparison of the SMS control with OS and SOS controls with $K_{S}$ having three corner points. Model parameters used are given in Table 2.

biomass productions), often mentioned in the literature as a relevant tool for irrigation planning (see e.g. [3]). Other criterion could have been considered balancing between biomass production and water consumption, but that would require to choose weights in the mixed penalization or precise water pricing. Crop-water production functions provide more intrinsic information about the crop irrigation, the cost-benefit analysis being left to the decision-makers.

\section{Conclusion}

We have introduced a simple crop irrigation model in order to study optimal irrigation scheduling using mathematical analysis. We have first shown, using a comparison tool, that the state constraint of this model is never activated for the optimal control problem solutions.

Moreover, we have demonstrated that, under water scarcity, an optimal trajectory has to reach as fast as possible the domain for which the relative humidity is below or equal to the threshold of maximal crop transpiration, and then to maintain it in this domain until the harvesting time. However, due to water scarcity, it has to be strictly below this threshold at some stage. We have then proved that the optimal strategy consists of irrigating once but with possible multiple steps ("SMS strategy") and not necessarily with a single shot ("OS strategy") as commonly used in practice. Moreover, we have shown that when the water-stress functions do not present any corner in between their extreme values, the SMS strategy has at most one step which is necessary at the maximal crop transpiration threshold (that we called "SOS strategy"). The SOS strategy is simpler to apply than the SMS one (with more than one step) as the soil moisture has simply to be maintained at the transpiration threshold until the water quota is reached. A remarkable feature is that the structure of the optimal strategy does not depend on the radiation interception efficiency function (although optimal trigger threshold and step values do rely on this function). As the considered stress functions are piecewise linear, we have used the non-smooth Maximum Principle to obtain relatively simple strategies, relying on the single determination of singular arcs. Paradoxically, the consideration of smooth stress functions would have led to optimal strategies without a particular structure easy to characterize and to implement as the SMS (and SOS) ones.

We have then compared the three control strategies: the open-loop one-shot (OS), commonly used in practice, the feedback saturated one-shot (SOS), that could exhibit a singular arc, and the more sophisticated feedback strategy with multiple increasing shots (SMS), that could exhibit several singular arcs. We have shown numerically the superiority of this last strategy. We guess that the SMS strategy (that coincides with the SOS one for simple water-stress functions) could also be the best one in real situations. This would be a promising result since SMS/SOS irrigation schemes are not such intuitive control strategies, that could also be tested on simulations of more detailed models. This shall be the matter of future work. 


\section{Acknowledgments}

The authors thank \#DigitAg Convergence Institute for the support of the postdoctoral grant of N. Kalboussi, carried out at MISTEA and ITAP labs, and the AVERROES program for the funding of the PhD grant of K. Boumaza. This work has been achieved in the framework of the euro-Mediterranean network TREASURE (for Treatment and Sustainable Reuse of Effluents in semiarid climates). The authors are very grateful to O. Cots for exchanging about the numerical aspects, and thank B. Cheviron, J. Harmand and T. Simonneau for fruitful discussions.

\section{References}

[1] Bertrand, N., Roux, S., Forey, O., Guinet M. And Wery, J., Simulating plant water stress dynamics in a wide range of bi-specific agrosystems in a region using the BISWAT model, European Journal of Agronomy, vol. 99, 116-128, 2018.

[2] Bonnans, F., Giorgi, D., Heymann, B., Martinon, P. and Tissot, O. Bocop HJB 1.0.1 - User Guide. Technical Report INRIA 0467, 2015.

[3] Brumbelow, K. And Georgakakos, A. Determining crop-water production functions using yield-irrigation gradient algorithms. Agricultural Water Management, vol. 87, 151-161, 2007.

[4] Clarke, F., Functional Analysis, Calculus of Variations and Optimal Control, Springer 2013.

[5] Cheviron, B., and Vervoort, R. W., Albasha, R., Dairon, R., Le Priol, C. And Mailhol, J., A framework to use crop models for multi-objective constrained optimization of irrigation strategies, Environmental Modelling \& Software, 86, 145-157, 2016.

[6] Faulwasser, T., Korda, M., Jones, C. And Bonvin, On turnpike and disspativity properties of continuous-time optimal control problems, Automatica, 81, 297-304, 2017.

[7] Hartl, R.F. And Feichtinger, G., A new sufficient condition for most rapid approach paths, Journal of Optimization Theory and Applications, Vol; 54 (2), pp. 402-411, 1987.

[8] Hermes, H. And Lasalle, J.P. Functional analysis and time optimal control, Mathematics in Science and Engineering, Vol. 56, Elsevier, 1969.

[9] Hiriart-Urruty, J.B. And Lemaréchal, C. Fundamentals of Convex Analysis, Springer 2001.

[10] Kalboussi, N., Roux, S., Boumaza, K., Sinfort, C. And Rapaport, A., About modeling and control strategies for scheduling crop irrigation. IFAC Workshop on Control Methods for Water Resource Systems CMWRS, Delft (Netherlands), IFAC-PapersOnLine, Vol. 52(23), pp. 43-48, 2019.

[11] Kloss, S., Pushpalatha, R., Kamoyo, K. J., Sch $\tilde{\mathrm{A}} \frac{1}{4} \mathrm{TzE}, \mathrm{N}$. , Evaluation of crop models for simulating and optimizing deficit irrigation systems in arid and semi-arid countries under climate variability Water resources management 26(4), pp. 997-1014, 2012.

[12] Li, Z., Cournède, P.H. And Mailhol, J.C., Irrigation Optimization by Modeling of Plant-Soil Interaction, 2011 Proceedings of the Conference on Applied Simulation and Modelling, 2011.

[13] Lopes, S. O., Fontes, F. A. C. C., Pereira, R. M. S., De Pinho, MdR., And Gonçalves A. M., Optimal Control Applied to an Irrigation Planning Problem, Mathematical Problems in Engineering, 2016.

[14] Miele, A., Extremization of Linear Integrals by Green's Theorem, Optimization Techniques, Edited by G. Leitmann, Academic Press, New York, New York, pp. 69-98, 1962.

[15] Pelak, N., Revellia, R. And Porporato, A. A dynamical systems framework for crop models: Toward optimal fertilization and irrigation strategies under climatic variability, Ecological Modelling, Vol. 365, pp. 80-92, 2017.

[16] Rapaport, R. And CARtigny, P., Turnpike theorems by a value function approach, ESAIM Control, Optimizationa and Calculus of variations, 10, 123-141, 2004. 
[17] Shani, U, Tsur, Y And Zemel, A. Optimal dynamics irrigation schemes, Optimal Control, Applications and Methods, 25, 91-106, 2004.

[18] Schütze, N., Schmitz, G. H., New planning tool for optimal climate change adaption strategies in irrigation, Journal of Irrigation and Drainage Engineering, 136(12), pp. 836-846, 2010.

[19] Schütze, N., De Paly, M., And Shamir, U., Novel simulation-based algorithms for optimal open-loop and closed-loop scheduling of deficit irrigation systems, Journal of Hydroinformatics, Vol. 14(1), 136-151, 2011.

[20] Spence, M. And Starrett, D. Most Rapid Approach Paths in Accumulation Problems, International Economic Review, Vol. 16, pp. 388-403, 1975.

[21] Steduto, P., Hsiao, H., Raes D. And Fereres E., AquaCrop, The FAO crop model to simulate yield response to water: I. Concepts and underlying principles, Agronomy Journal, 101(3), 426-437, 2009.

[22] TrÉlat, E. And ZuazuA, E., The turnpike property in finite-dimensional nonlinear optimal control, Journal of Differential Equations, 258, 81-114, 2015.

[23] Vico, G. And Porporato, A., Probabilistic description of crop development and irrigation water requirements with stochastic rainfall, Water Resources Research, 49, 3, 1466-1482, 2013.

[24] Allen, Richard G. and Pereira, Luis S. and Raes, Dirk and Smith, Martin et al., Crop evapotranspiration - Guidelines for computing crop water requirements - FAO Irrigation and drainage paper 56, Fao, Rome, 1998.

[25] Vinter, R. Optimal Control, Systems \& Control: Foundations \& Applications, Birkhäuser, 2000.

[26] Walter, W. Ordinary differential equations, Springer, 1998. 